\title{
Giant Acceleration of Free Diffusion by Use of Tilted Periodic Potentials
}

\author{
P. Reimann, ${ }^{1}$ C. Van den Broeck, ${ }^{2}$ H. Linke, ${ }^{3,4}$ P. Hänggi, ${ }^{1}$ J. M. Rubi, ${ }^{5}$ and A. Pérez-Madrid ${ }^{5}$ \\ ${ }^{1}$ Universität Augsburg, Institut für Physik, Universitätsstrasse 1, D-86135 Augsburg, Germany \\ ${ }^{2}$ Limburgs Universitair Centrum, B-3590 Diepenbeek, Belgium \\ ${ }^{3}$ School of Physics, University of New South Wales, Sydney 2051, Australia \\ ${ }^{4}$ Department of Physics, University of Oregon, Eugene, Oregon 97403-1274 \\ ${ }^{5}$ Departament de Fisica Fonamental, Facultat de Fisica, Universitat de Barcelona, Diagonal 647, E-08028 Barcelona, Spain
}

(Received 27 February 2001; published 18 June 2001)

\begin{abstract}
The effective diffusion coefficient for the overdamped Brownian motion in a tilted periodic potential is calculated in closed analytical form. Universality classes and scaling properties for weak thermal noise are identified near the threshold tilt where deterministic running solutions set in. In this regime the diffusion may be greatly enhanced, as compared to free thermal diffusion with, for a realistic experimental setup, an enhancement of up to 14 orders of magnitude.
\end{abstract}

DOI: $10.1103 /$ PhysRevLett.87.010602

Thermal diffusion in a tilted periodic potential plays a prominent role in Josephson junctions [1], rotating dipoles in external fields [2], superionic conductors [3], charge density waves [4], synchronization phenomena [5], diffusion on crystal surfaces [6], particle separation by electrophoresis [7], and biophysical processes such as intracellular transport [8], to name just a few [9]. Also the Brownian motion in a "traveling periodic potential" $V(x-v t)$ can be readily mapped onto a static tilted periodic potential [10].

In many cases of interest, the diffusion can be modeled as overdamped Brownian motion in 1D:

$$
\eta \dot{x}(t)=-V^{\prime}(x(t))+F+\sqrt{2 \eta k T} \xi(t),
$$

where $\eta$ is the viscous friction coefficient (static mobility), $V(x)$ is a periodic potential,

$$
V(x+L)=V(x)
$$

$F$ is a static "tilting force," and $k$ is Boltzmann's constant. The thermal fluctuations at temperature $T$ are modeled $[9,11]$ by the unbiased $\delta$-correlated Gaussian noise $\xi(t)$.

The first basic quantity of interest is the particle current $\langle\dot{x}\rangle:=\lim _{t \rightarrow \infty}\langle x(t)\rangle / t$. Its analytical solution [see Eq. (7) below] goes back to Stratonovich [12] and has subsequently been rederived many times [9]. In this Letter, the quantity of foremost interest is the effective diffusion coefficient

$$
D:=\lim _{t \rightarrow \infty} \frac{\left\langle x^{2}(t)\right\rangle-\langle x(t)\rangle^{2}}{2 t} .
$$

For $V^{\prime}(x) \equiv 0$ and arbitrary $F$, the diffusion coefficient is given by Einstein's result $D_{0}:=k T / \eta$, whereas for $F=0$ and arbitrary $V(x)$, an analytical prediction for $D$ is due to [13]. In this Letter we derive an analytical formula for $D$ when both $V(x)$ and $F$ are arbitrary, analogous to Stratonovich's landmark result for $\langle\dot{x}\rangle$. Specifically, near the threshold tilt where deterministic running solutions set in, we find that diffusion is greatly enhanced and that it obeys a specific universal scaling relation.
PACS numbers: 05.40.-a, 02.50.Ey, 05.60.-k

Our starting point is the following exact expressions for the particle current and for the diffusion coefficient:

$$
\begin{gathered}
\langle\dot{x}\rangle=\frac{L}{\left\langle t\left(x_{0} \rightarrow x_{0}+L\right)\right\rangle}, \\
D=\frac{L^{2}}{2} \frac{\left\langle t^{2}\left(x_{0} \rightarrow x_{0}+L\right)\right\rangle-\left\langle t\left(x_{0} \rightarrow x_{0}+L\right)\right\rangle^{2}}{\left\langle t\left(x_{0} \rightarrow x_{0}+L\right)\right\rangle^{3}},
\end{gathered}
$$

where $x_{0}$ is an arbitrary reference point, $\left\langle t^{n}(a \rightarrow b)\right\rangle$ is the $n$th moment of the first passage time from $a$ to $b>a$ for a stochastic trajectory obeying (1), and where it is assumed that $F>0$ in order to keep those moments finite. Such relations have been previously proposed for certain random processes in discrete space $[14,15]$ and have been anticipated without proof in [16] also for the present continuous dynamics (1). A proof will be given at the end of this Letter (see also [17]).

For the dynamics (1), the moments of the first passage time are given by the well-known closed analytical recursion (see, e.g., section 7 in [11], and further references therein)

$$
\begin{aligned}
\left\langle t^{n}(a \rightarrow b)\right\rangle= & \int_{a}^{b} d x \int_{-\infty}^{x} \frac{d y}{D_{0}} n\left\langle t^{n-1}(y \rightarrow b)\right\rangle \\
& \times \exp \{[V(x)-V(y)-(x-y) F] / k T\}
\end{aligned}
$$

for $n=1,2, \ldots$, with $\left\langle t^{0}(y \rightarrow b)\right\rangle:=1$. By introducing (6) into (4) and (5), one finds, after somewhat tedious manipulations, the result

$$
\begin{gathered}
\langle\dot{x}\rangle=\frac{1-e^{-L F / k T}}{\int_{x_{0}}^{x_{0}+L} \frac{d x}{L} I_{+}(x)}, \\
D=D_{0} \frac{\int_{x_{0}+L}^{x_{0}+\frac{d x}{L}} I_{+}^{2}(x) I_{-}(x)}{\left[\int_{x_{0}}^{x_{0}+L} \frac{d x}{L} I_{+}(x)\right]^{3}},
\end{gathered}
$$

where we have introduced

$$
I_{ \pm}(x):=\int_{0}^{L} \frac{d y}{D_{0}} e^{\{ \pm V(x) \mp V(x \mp y)-y F\} / k T} .
$$


The so far excluded case $F<0$ can be readily reduced to an equivalent dynamics with $F>0$, yielding exactly the same result [(7) and (8)]. Finally, the case $F=0$ follows by continuation, in agreement with [13]. Note that (2) implies $I_{ \pm}(x)=I_{ \pm}(x+L)$ and hence the choice of $x_{0}$ is indeed irrelevant in (7) and (8). Further, Einstein's relation $D=D_{0}$ is readily recovered by observing that $I_{ \pm}(x)=$ const in the special case $V^{\prime}(x) \equiv 0$.

Equation (7) is Stratonovich's exact expression for the particle current [12], while the corresponding general formula for the diffusion coefficient (8) is the first central result of this Letter. Figure 1 exemplifies its excellent agreement with accurate numerical simulations, while an approximate result from [18] captures only the qualitative behavior. The most interesting feature in Fig. 1 is the peak near the "critical tilt" $F_{c}$, which gets more and more pronounced as $k T$ (or $D_{0}=k T / \eta$ ) decreases. This brings us to our second main preoccupation, namely, the case of weak noise $\left(k T \ll L F_{c}\right)$ in combination with a tilt $F$ close to the "critical" threshold value $F_{c}$ beyond which deterministically running solutions appear in (1). The resulting diffusion depends crucially on the form of the potential around its "dynamical bottleneck" $x_{c}$. Without loss of generality, we focus on $x_{c}=0$ and we assume the following small $x$ behavior:

$$
V(x)-x F=-\mu x|x|^{q-1}-\epsilon x,
$$

with $\mu>0, q>1$ (to guarantee differentiability at $x=0$ ), and small $\epsilon:=F-F_{c}$. In the remainder of the

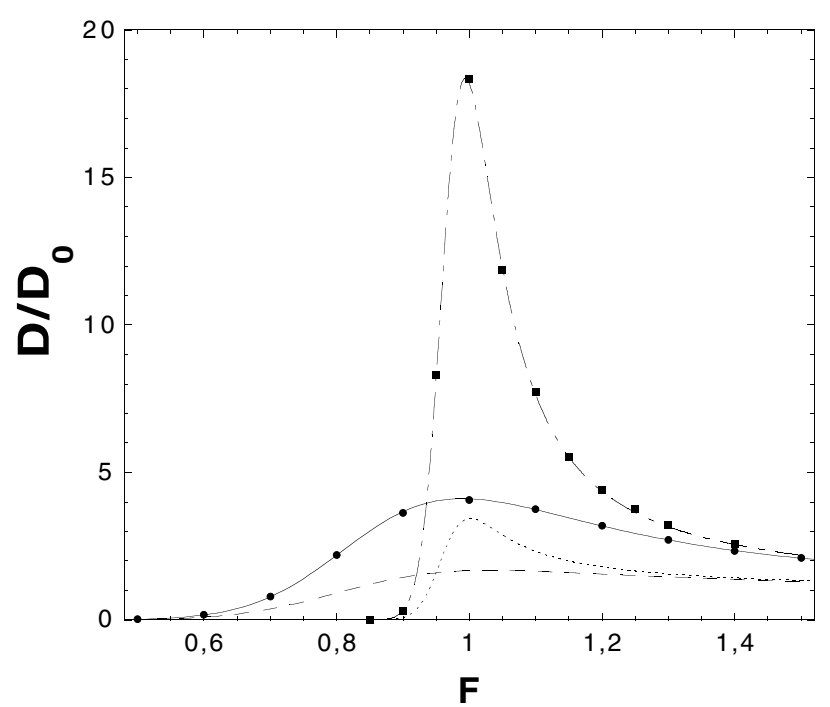

FIG. 1. Diffusion coefficient (3) versus the tilt $F$ for the stochastic dynamics (1) with a sinusoidal periodic potential $V(x)=$ $U_{0} \sin (2 \pi x / L)$. Using dimensionless units, the parameter values are $\eta=U_{0}=1, L=2 \pi$, and $k T=D_{0}=0.1$. Note that the critical tilt [onset of deterministically running solutions in (1)] occurs at $F=F_{c}=1$. Solid line: analytical prediction (8). Filled dots: numerical simulations with an estimated relative uncertainty of 0.01. Dashed line: analytical approximation $D=k T d\langle\dot{x}\rangle / d F$ from [18]. Dash-dotted line, filled squares, and dotted line: same as solid line, filled dots, and dashed line, respectively, but now for $k T=D_{0}=0.01$. interval $[-L / 2, L / 2]$, the "total potential" $V(x)-x F$ in (1) is assumed to be strictly monotonically decreasing (i.e., "tilted to the right" with $F_{c}>0$ ). Outside $[-L / 2, L / 2]$ the behavior of $V(x)$ is fixed by the periodicity (2). Note that $q=3$ in the generic case [e.g., an analytic $V(x)$ such as in Fig. 1]. Other $q$ values can be readily realized experimentally by tailoring the form of $V(x)$.

To get an intuitive feeling about the role of the exponent $q$, it is instructive to study the deterministic motion (1) in a critical $(\epsilon=0)$ potential (10) extending over the entire $x$ axis, i.e., $\dot{x}(t)=\alpha|x(t)|^{q-1}$ with $\alpha:=\mu q / \eta>0$. A straightforward calculation then shows that it takes an infinite amount of time to travel from any $x_{i}<0$ to $x_{f}=0$ when $q \geq 2$, while a finite time is sufficient for $1<q<2$. On the other hand, finite time suffices to go from $x_{i}=-\infty$ to any $x_{f}<0$ for $q>2$, while this traveling time diverges for $q \leq 2$. Complementary results are found for the traveling times in the region $x \geq 0$. We thus expect that for $q>2$, with sufficiently small (but finite) $k T$ and sufficiently small $\epsilon$, the motion of the particle is dominated by the passage through the bottleneck region in the vicinity of $x=0$, where the potential is well approximated by the form given in (10). On the other hand, for $2 \geq q>1$, the form of $V(x)$ outside a small neighborhood of $x=0$ is also expected to come into play.

With this insight in mind, we turn to the evaluation of (8) when $q>2$. First, it is convenient to make the special choice $x_{0}=-L / 2$. Second, in the vicinity of $x=0$ the approximation (10) can be introduced into (9). Third, due to our above considerations we can extend this approximation to the entire interval $[-L / 2, L / 2]$ and finally extend this integration domain to $[-\infty, \infty]$ without notably changing the values of the integrals in (8). Closer inspection shows that this approximation in fact becomes asymptotically exact as $\epsilon$ and $k T$ tend to zero. In this way, one obtains for $q>2$ the result

$$
D=D_{0}\left(\frac{L^{q} \mu}{k T}\right)^{2 / q} \frac{\int d x K^{2}(x, \gamma) K(-x, \gamma)}{\left[\int d x K(x, \gamma)\right]^{3}},
$$

where integration limits $\pm \infty$ have been omitted and

$$
\begin{gathered}
\gamma:=\epsilon /\left[\mu^{1 / q}(k T)^{1-1 / q}\right], \\
K(x, \gamma):=\int_{0}^{\infty} d y e^{\left\{-x|x|^{q-1}+(x-y)|x-y|^{\mid-1}-\gamma y\right\}} .
\end{gathered}
$$

Note that both fractions in (11) as well as the "scaled tilt" $\gamma$ are dimensionless and that $K(x, \gamma)$ is a dimensionless function of its (dimensionless) arguments $x$ and $\gamma$. Hence, (11) has the form of a scaling law with a universal scaling function and specific critical exponents depending on $q>2$. The most remarkable feature is the divergence of $D / D_{0}$ when $k T$ tends to zero for any fixed $\gamma$ value, i.e., we recover (cf. Fig. 1) a huge enhancement of thermal diffusion. For $q=3$, the scaling function appearing in (11) is depicted in Fig. 2. From this plot it follows that the asymptotic scaling form (11) is already rather well satisfied for moderately small $k T$ values and that the enhancement of 


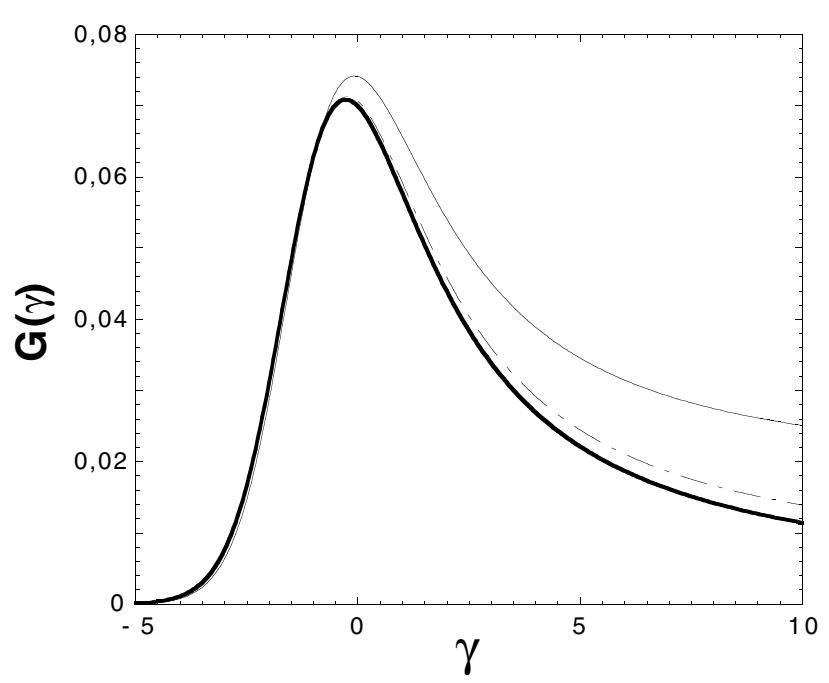

FIG. 2. Bold solid line: dimensionless scaling function $G(\gamma):=\int d x K^{2}(x, \gamma) K(-x, \gamma) /\left[\int d x K(x, \gamma)\right]^{3}$ in (11) for $q=3$ versus its dimensionless argument $\gamma$ from (12). Solid and dash-dotted lines: same as the respective lines in Fig. 1, but now plotted in the form $\left(D / D_{0}\right)\left(k T / L^{q} \mu\right)^{2 / q}$ [cf. (11)] versus $\gamma$ [cf. (12)] with $q=3$.

diffusion is most pronounced for $|\gamma| \leq \mathcal{O}$ (1). Similar behavior is recovered for any other $q>2$. In other words, the diffusion coefficient as a function of $F$ exhibits a pronounced "resonance" peak at $F=F_{c}$.

The basic physical mechanism behind this effect may be explained as follows. As discussed above, the noisy dynamics (1) is, for $\epsilon=0$ and small $k T$, dominated by the passage through the dynamical bottleneck at $x=0$ [cf. (10)]. Since $\epsilon=0$, a very small perturbation due to thermal noise is already sufficient to kick the particle across the point $x=0$. This small variation in comparison with an unperturbed particle is subsequently greatly enhanced by the further dynamical evolution. The result is a huge dispersion for a statistical ensemble of particles subjected to different realizations of the noise.

Finally, we briefly turn to the case $q \leq 2$. The region outside a small neighborhood of $x=0$ is then no longer negligible for the passage time from $-L / 2$ to $L / 2$, rendering the analysis more complicated. For simplicity, we restrict ourselves to the interesting situation of a potential given by (10) in the entire interval $[-L / 2, L / 2]$ with $2>q>4 / 3$, yielding the result

$$
D=D_{0}\left(\frac{L^{q} \mu}{k T}\right)^{3-4 / q} \frac{\int d x K^{2}(x, \gamma) K(-x, \gamma)}{\left[\left(\frac{k T}{L^{q} \mu}\right)^{2 / q-1} S(\gamma)+\frac{2^{q-1}}{q(2-q)}\right]^{3}},
$$

where $S(\gamma \geq 0):=0$ and

$$
\begin{aligned}
S(\gamma<0):= & \frac{2 \pi|\gamma / q|^{(2-q) /(q-1)}}{q(q-1)} \\
& \times \exp \left\{2(q-1)|\gamma / q|^{q /(q-1)}\right\} .
\end{aligned}
$$

Technical details as well as the discussion of other $q$ values and more complicated potentials $V(x)$ will be given elsewhere.
The salient difference of (14) in comparison with (11) is a competition between the two terms in the denominator on the right-hand side: for any fixed $\gamma$ value, the second term dominates when $k T$ becomes sufficiently small. Thus $D / D_{0}$ increases proportional to $(k T)^{4 / q-3}$, i.e., we find again a huge enhancement of thermal diffusion. More subtle is the behavior of (14) as a function of $\gamma$ for a small but fixed $k T$ value. For arbitrary positive as well as for moderately negative $\gamma$ values, it is still the second term in the denominator which dominates, and thus the $\gamma$ dependence of $D$ is governed by $\int d x K^{2}(x, \gamma) K(-x, \gamma)$. On the other hand, for large negative $\gamma$ values we can evaluate the latter integral by means of a saddle point approximation, yielding the result $S^{2}(\gamma) / 2$. Since $S(\gamma)$ from (15) increases very fast with decreasing $\gamma$, the right-hand side of (14) increases very fast as long as $\int d x K^{2}(x, \gamma) K(-x, \gamma)$ governs the $\gamma$ dependence. However, again due to this fast increase, the first summand in the denominator starts to compete with the second summand and ultimately takes over, leading to a decrease of $D$ proportional to $1 / S(\gamma)$. Thus a peak appears at a (negative) $\gamma$ value for which both terms in the denominator are of the same order of magnitude. The detailed quantitative calculation is straightforward and leads, for $2>q>4 / 3$, to the result

$$
\begin{gathered}
D\left(\gamma_{\max }\right)=\frac{2^{2-q} q(2-q)}{27} \frac{L^{q} \mu}{\eta}, \\
\gamma_{\max } \simeq-q\left[\frac{2-q}{2 q(q-1)} \ln \left(\frac{L^{q} \mu}{k T}\right)\right]^{1-1 / q} .
\end{gathered}
$$

Note that the maximal diffusion coefficient in (16) is independent of $k T$. In other words, the maximal enhancement of diffusion is even stronger than for $q>2$ [cf. (11)]. These predictions are confirmed by direct numerical evaluation of the exact formula (8) in Fig. 3.

These results can be applied, for instance, to the thermally induced diffusion of a particle that moves in a liquid under the action of gravitation along the rigid surface of a critically tilted periodic geometrical profile. For a spherical iron particle of $1 \mathrm{~mm}$ radius in water and a critically tilted profile that decreases by $1.5 \mathrm{~cm}$ per spatial period $L=10 \mathrm{~cm}$, one finds, at room temperature for the thermal diffusion coefficient, $D_{0} \simeq 2 \times 10^{-12} \mathrm{~cm}^{2} / \mathrm{s}$. From (11), with $q=3$, one finds at the critical tilt $D \simeq$ $5 \times 10^{-3} \mathrm{~cm}^{2} / \mathrm{s}$, i.e., an enhancement by about 9 orders of magnitude. For a specially tailored profile of the form (10) with $q=3 / 2$, one finds from (16) that $D \simeq 2 \times$ $10^{2} \mathrm{~cm}^{2} / \mathrm{s}$, i.e., the enhancement of thermal diffusion is improved by another 5 orders of magnitude as compared to the case $q=3$ and should be easily observable on macroscopic length and time scales. An actual experimental realization is presently under construction.

We conclude with the promised proof of (4) and (5) for $F>0$ and an arbitrary $x_{0}$. To this end, we denote by $c$ an arbitrary point between $a$ and $b>a$. Then the first passage time $t(a \rightarrow b)$ can be decomposed into the time to travel from $a$ to $c$, plus the time to travel from $c$ to $b$. For 


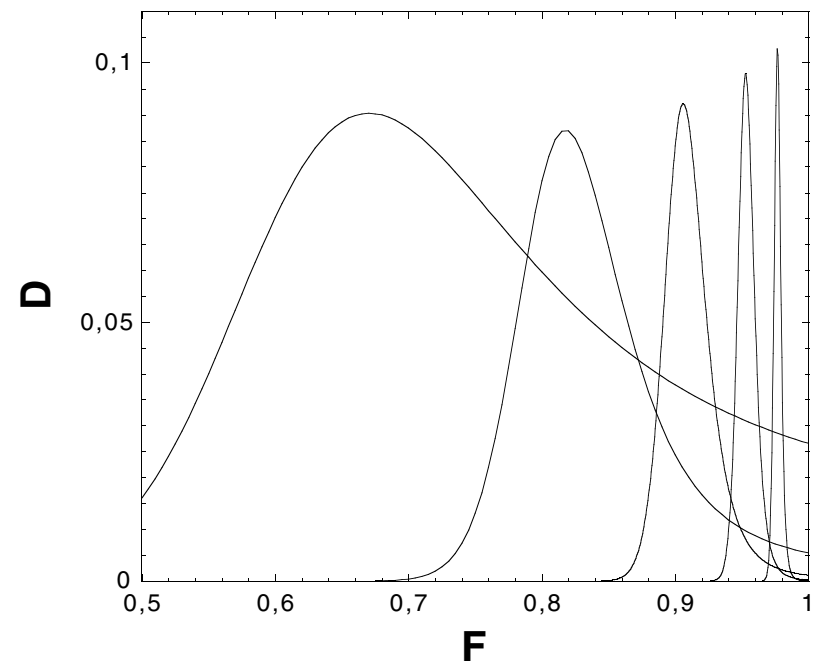

FIG. 3. Diffusion coefficient (8) versus the tilt $F$ for a potential $V(x)=V(x+L)$ defined by (10) for $x \in[-L / 2, L / 2]$. Using dimensionless units, the parameter values are $q=3 / 2, \eta=1$, $L=2, \mu=1, F_{c}=1$. The five curves, with sharper peaks corresponding to lower temperatures, represent the following values of $k T=D_{0}: 3 \times 10^{-2}, 10^{-2}, 10^{-3}, 10^{-4}, 10^{-5}$. The theoretically predicted peak height for asymptotically small $k T$ from (16) is $0 . \overline{1}$.

a white noise driven process (1), the latter two times are statistically independent of each other [19] and all statistical properties of $t(a \rightarrow b)$ are exactly the same as those of $t(a \rightarrow c)+t(c \rightarrow b)$. As a consequence, $\langle t(a \rightarrow b)\rangle=$ $\langle t(a \rightarrow c)\rangle+\langle t(c \rightarrow b)\rangle$ and analogously for the first passage time dispersion $\left\langle\Delta t^{2}(a \rightarrow b)\right\rangle:=\left\langle t^{2}(a \rightarrow b)\right\rangle-$ $\langle t(a \rightarrow b)\rangle^{2}$. Further, $t\left(x_{0} \rightarrow x_{0}+l L\right)$ is statistically equivalent to a sum of $l$ independent, random variables, $t\left(x_{0} \rightarrow x_{0}+L\right), \ldots, t\left(x_{0}+(l-1) L \rightarrow x_{0}+l L\right)$, and, due to the periodicity (2), they are identically distributed. Invoking the central limit theorem, the distribution of the first passage times $t\left(x_{0} \rightarrow x_{0}+l L\right)$ thus approaches for large $l$ a Gaussian distribution with mean value $l\left\langle t\left(x_{0} \rightarrow\right.\right.$ $\left.\left.x_{0}+L\right)\right\rangle$ and variance $l\left\langle\Delta t^{2}\left(x_{0} \rightarrow x_{0}+L\right)\right\rangle$.

Next, we introduce "coarse-grained states" $\left\{x_{m}:=x_{0}+\right.$ $m l L\}_{m=-\infty}^{\infty}$, where $l$ is a large but fixed integer [20]. The process $x(t)$ is said to be in a certain "state" from the instant it hits the associated point $x_{m}$ until the moment it hits one of the adjacent points $x_{m \pm 1}$. It follows that the diffusion coefficient $D$ is identical for the original process $x(t)$ and its coarse-grained counterpart, due to the long-time limit in (3), and similarly for the current $\langle\dot{x}\rangle$. Next we note that "backward transitions" $x_{m} \mapsto x_{m-1}$ are suppressed by a Boltzmann factor $\exp \{-l L F / k T\}$ compared to $x_{m} \mapsto$ $x_{m+1}$ and therefore are negligible for sufficiently large $l$. The remaining "forward transitions" between neighboring "states" $x_{m}$ and $x_{m+1}$ are identically distributed random events with a probability distribution which is identical to the first passage time distribution for the original process $x(t)$. On the other hand, we have seen above that for sufficiently large $l$ this distribution is completely fixed by $\left\langle t\left(x_{0} \rightarrow x_{0}+L\right)\right\rangle$ and $\left\langle\Delta t^{2}\left(x_{0} \rightarrow x_{0}+L\right)\right\rangle$. Thus, if the latter two quantities are the same for two processes (1) then $\langle\dot{x}\rangle$ and $D$ will also be the same in the two cases. Consequently, it is sufficient to prove (4) and (5) for the special case that $V^{\prime}(x) \equiv 0$. In this case, $\langle\dot{x}\rangle=F / \eta, D=D_{0}$, and the evaluation of $\left\langle t\left(x_{0} \rightarrow x_{0}+L\right)\right\rangle$ and $\left\langle\Delta t^{2}\left(x_{0} \rightarrow\right.\right.$ $\left.\left.x_{0}+L\right)\right\rangle$ according to (6) is straightforward. As a result, one sees that (4) and (5) are indeed fulfilled.

This work was supported by the exchange program of the Deutscher Akademischer Austauschdienst (P. R., P. H.) and the Acciones Integradas Hispano-Alemanas under HA1999-0081 (J.M.R., A. P.-M.), the Program in InterUniversity Attraction Poles of the Belgian Government (C. V.d.B.), the Australian Research Council (H. L.), DFGSachbeihilfe HA1517/13-2, and the Graduiertenkolleg GRK283 (P.R., P.H.).

[1] A. Barone and G. Paterno, Physics and Applications of the Josephson Effect (Wiley, New York, 1982).

[2] D. Reguera, J. M. Rubi, and A. Pérez-Madrid, Phys. Rev. E 62, 5313 (2000).

[3] P. Fulde, L. Pietronero, W. R. Schneider, and S. Strässler, Phys. Rev. Lett. 35, 1776 (1975).

[4] G. Gruner, A Zawadowski, and P. M. Chaikin, Phys. Rev. Lett. 46, 511 (1981).

[5] W. C. Lindsey, Synchronization Systems in Communication and Control (Prentice-Hall, Englewood Cliffs, NJ, 1972).

[6] J. W. M. Frenken and J. F. Van der Veen, Phys. Rev. Lett. 54, 134 (1985); B. Pluis et al., ibid. 59, 2678 (1987).

[7] A. Ajdari and J. Prost, Proc. Natl. Acad. Sci. U.S.A. 88, 4468 (1992); G. I. Nixon and G. W. Slater, Phys. Rev. E 53, 4969 (1996).

[8] For a review, see P. Reimann, cond-mat/0010237.

[9] H. Risken, The Fokker-Planck Equation (Springer, Berlin, 1984).

[10] M. Borromeo and F. Marchesoni, Phys. Lett. A 249, 8457 (1998); C. Van den Broeck, Europhys. Lett. 46, 1 (1999).

[11] P. Hänggi, P. Talkner, and M. Borkovec, Rev. Mod. Phys. 62, 251 (1990).

[12] R. L. Stratonovich, Radiotekh. Elektron. 3, 497 (1958).

[13] S. Lifson and J.L. Jackson, J. Chem. Phys. 36, 2410 (1962). The same result follows from the fluctuationdissipation relation $D=k T d\langle\dot{x}\rangle /\left.d F\right|_{F=0}$.

[14] D. R. Cox, Renewal Theory (Methuen \& Co., London, 1962), Chap. 5, Eqs. (14) and (16).

[15] M. Khantha and V. Balakrishnan, Pramana 21, 111 (1983); C. Van den Broeck, A Glimpse into the World of Random Walks, edited by J. L. Munoz-Cobo and F. Difilippo, Noise and Nonlinear Phenomena in Nuclear Systems (Plenum, New York, 1989).

[16] P. Reimann and C. Van den Broeck, Physica (Amsterdam) 75D, 509 (1994).

[17] B. Lindner, M. Kostur, and L. Schimansky-Geier, Fluct. Noise Lett. 1, R25 (2001).

[18] G. Constantini and F. Marchesoni, Europhys. Lett. 48, 491 (1999).

[19] This is so because (1) is a strong Markov process, and first passage times are Markov stopping times.

[20] The $t \rightarrow \infty$ limit [e.g., in (3)] is associated with $m \rightarrow \infty$, not with $l \rightarrow \infty$. 